\title{
High-Efficiency Deflection of High-Energy Protons through Axial Channeling in a Bent Crystal
}

\author{
Walter Scandale, ${ }^{1}$ Alberto Vomiero, ${ }^{2}$ Stefano Baricordi, ${ }^{3}$ Pietro Dalpiaz, ${ }^{3}$ Massimiliano Fiorini, ${ }^{3}$ Vincenzo Guidi, ${ }^{3}$ \\ Andrea Mazzolari, ${ }^{3}$ Gianantonio Della Mea, ${ }^{4}$ Riccardo Milan, ${ }^{4}$ Giovanni Ambrosi, ${ }^{5}$ Bruna Bertucci, ${ }^{5}$ William J. Burger, ${ }^{5}$ \\ Paolo Zuccon, ${ }^{5}$ Gianluca Cavoto, ${ }^{6}$ Claudio Luci, ${ }^{6}$ Roberta Santacesaria,${ }^{6}$ Paolo Valente, ${ }^{6}$ Erik Vallazza, ${ }^{7}$ \\ Alexander G. Afonin, ${ }^{8}$ Yury A. Chesnokov, ${ }^{8}$ Vladimir A. Maisheev, ${ }^{8}$ Igor A. Yazynin, ${ }^{8}$ Alexander D. Kovalenko, ${ }^{9}$ \\ Alexander M. Taratin,,${ }^{9}$ Alexander S. Denisov, ${ }^{10}$ Yury A. Gavrikov, ${ }^{10}$ Yuri M. Ivanov,${ }^{10}$ Lyubov P. Lapina, ${ }^{10}$ \\ Liudmila G. Malyarenko, ${ }^{10}$ Vyacheslav V. Skorobogatov, ${ }^{10}$ Vsevolod M. Suvorov, ${ }^{10}$ Sergey A. Vavilov, ${ }^{10}$ \\ Davide Bolognini, ${ }^{11}$ Said Hasan, ${ }^{11}$ Aldo Mozzanica, ${ }^{11}$ and Michela Prest ${ }^{11}$ \\ ${ }^{1}$ CERN, European Organization for Nuclear Research, CH-1211 Geneva 23, Switzerland \\ ${ }^{2}$ INFM-CNR, Via Valotti 9, 25133 Brescia, Italy \\ ${ }^{3}$ INFN Sezione di Ferrara, Dipartimento di Fisica, Università di Ferrara, Via Saragat 1, 44100 Ferrara, Italy \\ ${ }^{4}$ INFN Laboratori Nazionali di Legnaro, Viale Università 2, 35020 Legnaro (PD), Italy \\ ${ }^{5}$ INFN Sezione di Perugia and Università degli Studi di Perugia, Dipartimento di Fisica, Via Pascoli, 06123 Perugia, Italy \\ ${ }^{6}$ INFN Sezione di Roma, Piazzale Aldo Moro 2, 00185 Rome, Italy \\ ${ }^{7}$ INFN Sezione di Trieste, Via Valerio 2, 34127 Trieste, Italy \\ ${ }^{8}$ Institute of High Energy Physics, Moscow Region, RU-142284 Protvino, Russia \\ ${ }^{9}$ Joint Institute for Nuclear Research, Joliot-Curie 6, 141980, Dubna, Moscow Region, Russia \\ ${ }^{10}$ Petersburg Nuclear Physics Institute, 188300 Gatchina, Leningrad Region, Russia \\ ${ }^{11}$ Università dell'Insubria, via Valleggio 11, 22100 Como, Italy, and INFN Sezione di Milano, via Celoria 16, 20133 Milan, Italy
} (Received 30 May 2008; published 16 October 2008)

\begin{abstract}
Beam deflection due to axial channeling in a silicon crystal bent along the $\langle 111\rangle$ axis was observed with $400 \mathrm{GeV} / c$ protons at the CERN Super Proton Synchrotron. The condition for doughnut scattering of protons by the atomic strings of the crystal was attained. Such a condition allowed one to observe a beam deflection of $50 \mu \mathrm{rad}$ with about $30 \%$ efficiency. The contribution of hyperchanneled states of protons to the observed beam deflection was less than $2 \%$ according to simulation results.
\end{abstract}

Planar channeling consists of transverse confinement of a charged particle motion between two crystallographic planes [1]. For a bent crystal, channeled particles follow the shape of deformed atomic planes and are deflected. Deflection of high-energy positively charged particles due to planar channeling in a bent crystal is a well-known effect, whose potential has been widely exploited for beam steering in high-energy accelerators [2-6].

As a particle traverses the crystal with a small angle $\psi_{0}$ to one of the main crystallographic axes, the regime of axial channeling (AC) is realized [1]. In this case the particle motion is governed by the potential of the lattice of atomic strings averaged along the axis [1]. The angular acceptance of AC demands the alignment of the particle trajectory with a string $\psi_{0} \leq \psi_{1}$, where $\psi_{1}$ is the critical angle for axial channeling.

Two types of states, "hyperchanneling" and "doughnut scattering," are realized for particles under axial channeling. The first one is the confinement of particles bounded up with a single axial channel. However, the potential barrier separating the axial channels formed by neighboring atomic strings is rather low; thus, only a small fraction of the particles can be captured into hyperchanneling states even for perfect alignment of the crystal axis with the beam. For silicon crystal, such a potential barrier is about $6 \mathrm{eV}$ for the $\langle 110\rangle$ direction and only $1 \mathrm{eV}$ for the $\langle 111\rangle$ direction. Particles with a transverse energy higher than the potential barrier wander in the lattice of atomic strings changing the transverse momentum direction in collisions with the strings. For particles with an angle smaller than $\psi_{1}$, a uniform distribution of the transverse momentum directions is reached after traversing a distance $\lambda$ (equalization length) [1]. The process of scattering by atomic strings that leads to such a distribution is called doughnut scattering. For incident angles larger than $\psi_{1}$, multiple scattering of particles by atomic strings leads to a partial equalization of the transverse momentum directions resulting in an asymmetric angular distribution of particles (in the form of an arc around the axis direction).

Indeed, $\mathrm{AC}$ in a bent crystal would represent an alternative to planar channeling for particle steering. The deflection of positively charged particles axially channeled in a bent crystal was first shown in Ref. [7] by simulation using a binary-collision model. The observed wide spectrum of deflection angles from zero to the full bending angle of the crystal $\alpha$ was caused by channeled particles wandering among bent axial channels.

With some limitations on the crystal bending, doughnut scattering does not significantly smear out the trajectories transversely; i.e., the ensemble of the channeled particles proceeds along the bent axes and undergoes deflection by the crystal bending angle. Thus, deflection of axially chan- 
neled particles is possible not only due to the bound states of hyperchanneling but also due to the unbound states of doughnut scattering regime. Indeed, the mechanism of doughnut scattering would allow one to steer a considerable part of the beam.

The condition for particle deflection in a bent crystal due to doughnut scattering was first formulated in Ref. [8]. Pioneering experiments $[8,9]$ actually did not observe the beam deflection due to AC. Such experiments mainly highlighted a strong feed-in of particles into skew planar channels stretched to the side opposite to that of bending. A small beam fraction deflected up to the full crystal bending angle in the experiment described in Ref. [9] was later interpreted by simulation as partly generated by hyperchanneled particles [10].

Indeed, a sufficient condition for deflection of particles through doughnut scattering in AC was formulated in Ref. [11] and here reported:

$$
\begin{gathered}
k=k_{1} k_{2}<1, \quad \text { with } k_{1}=\frac{\lambda}{R \psi_{1}} \quad \text { and } \quad k_{2}=\frac{L}{R \psi_{1}}, \\
\psi_{1}=\sqrt{\frac{4 Z_{1} Z_{2} e^{2}}{p v d}}, \quad \lambda\left(\psi_{1}\right)=\frac{4}{\pi^{2} N d a \psi_{1}},
\end{gathered}
$$

where $R$ and $L$ are the radius of curvature and length of the crystal, respectively, $Z_{1}$ and $Z_{2}$ are the atomic numbers of the incident particle and the crystal atom, respectively, $p$ and $v$ are the momentum and velocity, respectively, of the particle, $d$ is the interatomic spacing in the string, $N$ is the atomic density in the crystal, and $a$ is the screening length for the particle-atom potential $(a=0.194 \AA$ for $\mathrm{Si})$. Equation (1) expresses not only a limitation to the bending of the crystal along the equalization length $k_{1}<1$ but also limits the full bending angle of the crystal $(\alpha=L / R)$.

The experiment in Ref. [8] was performed with silicon crystals bent along the $\langle 111\rangle$ axes through a three-point bender, interacting with $12 \mathrm{GeV} / c$ secondary particles (protons and $\pi^{+}$) from the CERN Proton Synchrotron. In these conditions $\psi_{1}=0.12 \mathrm{mrad}$ and $\lambda=7.42 \mu \mathrm{m}$. For the case analyzed in Ref. [8], the crystal length of $2 \mathrm{~cm}$ and the bend angle of $20 \mathrm{mrad}$, the length of the crystal bent part should be about $1.5 \mathrm{~cm}$, which gives the average bend radius $R=0.75 \mathrm{~m}$ and the value of $k=13.8$. Thus, the condition (1) was not satisfied. Really, for small angles of particle orientations with the $\langle 111\rangle$ axis (see the first part of Fig. 9 in [8]), there are no particles, which were deflected by the bend angle. The angular distribution is like the "swallow tail," which begins from the incident beam direction and is directed to the bend side. The distribution was generated by particles, which were captured into the skew (110) planes at the crystal entrance and then were dechanneled.

A silicon crystal bent along the $\langle 110\rangle$ axes was used in the experiment [9] with a beam of $450 \mathrm{GeV} / c$ protons. The channeling parameters were $\psi_{1}=21.6 \mu \mathrm{rad}$ and $\lambda=$
$50.3 \mu \mathrm{m}$. The part of the crystal that was bent was $3 \mathrm{~cm}$ and the bending angle $3.1 \mathrm{mrad}$ - the average bend radius $R=9.7 \mathrm{~m}$ and the parameter $k=34.6$. The condition (1) for the deflection of protons due to doughnut scattering was not fulfilled here, too.

This work presents the experimental results obtained at the CERN Super Proton Synchrotron about the deflection of $400 \mathrm{GeV} / c$ protons mainly due to doughnut scattering along the $\langle 111\rangle$ axes of a bent silicon crystal.

A $70 \times 2 \times 0.5 \mathrm{~mm}^{3}$ silicon strip with the largest faces parallel to the (110) crystallographic planes and with its side faces cut parallel to the (111) planes was designed for the purpose [12]. The crystal was placed in a vertical position in such a way that the proton beam entered the crystal through its side face nearly parallel to the large faces (see Fig. 1). Thus, the $\langle 111\rangle$ axis direction, which is normal to the side crystal faces, became nearly aligned with the beam direction. The crystal was mechanically bent (primary bending) at the edges of the strip by a holder, which imparted the crystal a secondary bending (called anticlastic). The anticlastic curvature produced by the bending device along the crystal width was used for beam deflection in the horizontal plane. The usage of anticlastic deformation allows one to design relatively short bent crystals [5], and the favorable connection between primary and secondary curvatures (of the order of Poisson's coefficient) permits one to achieve easily very small angles of the crystal bend. Such crystals are being studied for the LHC collimation system as a primary collimator to throw the beam halo particles onto the absorber far from its edge due to the efficient deflection of particles at planar channeling or volume reflection in a bent crystal.

The bending angle was $\alpha=50 \mu \mathrm{rad}$ and the corresponding bending radius $R=40 \mathrm{~m}$. The critical angle for $\mathrm{AC}$ of $400 \mathrm{GeV} / c$ protons along the $\langle 111\rangle$ direction is $\psi_{1}=20.7 \mu \mathrm{rad}$ (i.e., about twice the critical angle for planar channeling), the equalization length $\lambda=43 \mu \mathrm{m}$, and $k=0.126$. Thus, contrary to the previous experiments [8,9], the condition (1) was satisfied in our case due to using a short bent crystal. This gave us the possibility to observe the deflection of particles due to doughnut scattering in a bent crystal.

The experimental setup was improved with respect to the one described in Ref. [13]. The deflection angles of the protons due to interaction with the crystal were measured to be silicon microstrip detectors with very good angular resolution $(3 \mu \mathrm{rad})$. The goniometer was upgraded with an additional rotational (cradle) stage, which allowed the orientation of the crystal with respect to a horizontal axis. The cradle stage has $\pm 6.3^{\circ}$ total range, $0.25 \mu \mathrm{rad}$ resolution, about $1 \mu \mathrm{rad}$ repeatability, and $2 \mu \mathrm{rad}$ accuracy. The angular divergence of the primary beam along the horizontal and vertical planes were measured as $\sigma_{x}=$ $(9.27 \pm 0.06) \mu \mathrm{rad}$ and $\sigma_{y}=(5.24 \pm 0.03) \mu \mathrm{rad}(\mathrm{rms}$ values), respectively. 


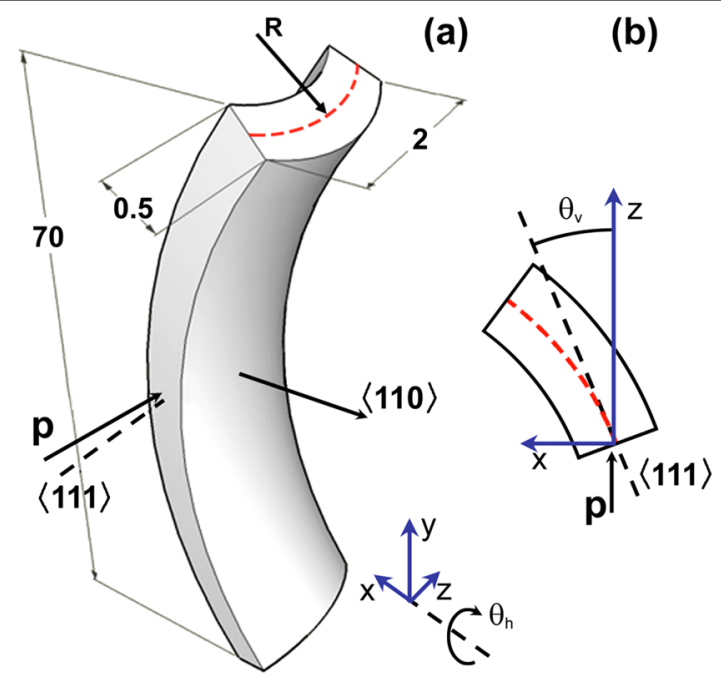

FIG. 1 (color online). (a) Schematic picture of a silicon crystal bent by anticlastic deformation along the $\langle 111\rangle$ axis. (b) Crosssectional view of the crystal. Dimensions are expressed in millimeters. $\theta_{v}$ and $\theta_{h}$ are the orientation angles of the $\langle 111\rangle$ axis relative to the proton beam direction.

After the installation, an angular scan of the crystal around the vertical axis $\left(\theta_{v}\right)$ was done to orient the (110) planes parallel to the beam direction. Then angular scans were performed at different cradle angles $\left(\theta_{h}\right)$ to reach the best possible alignment of the $\langle 111\rangle$ axis with the beam, according to the procedure described in Ref. [14].

Figure 2 shows the beam intensity distributions as a function of the particle deflection in the horizontal $\left(\theta_{x}\right)$ and vertical directions $\left(\theta_{y}\right)$ due to the interaction with the crystal at three different values of $\theta_{v}$. When the beam direction is far from the axial direction, the beam is undeflected, resulting in a spot, not shown in the figure, centered in $\left(\theta_{x} ; \theta_{y}\right)=(0 ; 0)$ with standard deviations close to the beam divergence quoted above. As $\theta_{v}$ approached the alignment condition, the distribution in Fig. 2(a) was observed. A large fraction of the particles was still undeflected, though part of the beam was deflected due to scattering by atomic strings and feeding into skew planar channels. The bottom spot is more intense than the top one because of a nonperfect alignment of the crystal with respect to the beam in the direction of $\theta_{h}$.

By increasing $\theta_{v}$, the distribution is further modified as shown in Fig. 2(b). Here only a small fraction of the beam remained undeflected. Axial channeling along the $\langle 111\rangle$ axis occurred and deflected the beam by the crystal bending angle, which was about $50 \mu \mathrm{rad}$. A fraction of the initial axially channeled particles leaked into skew planar channels because the $\langle 111\rangle$ axis is the intersection of several planes. Scattering with $\langle 111\rangle$ atomic strings may accidentally direct a particle parallel to one of the skew planes; then the crystal bending strengthens the stability of this planar motion. Deflection of particles due to channeling by the strongest (110) skew channels was clearly observed as two tails departing from the axial spot.

The best alignment condition is shown in Fig. 2(c). The undeflected part of the beam has completely disappeared, and the spot corresponding to axial channeling is the most populated by beam particles. A "swallow-tail" profile was

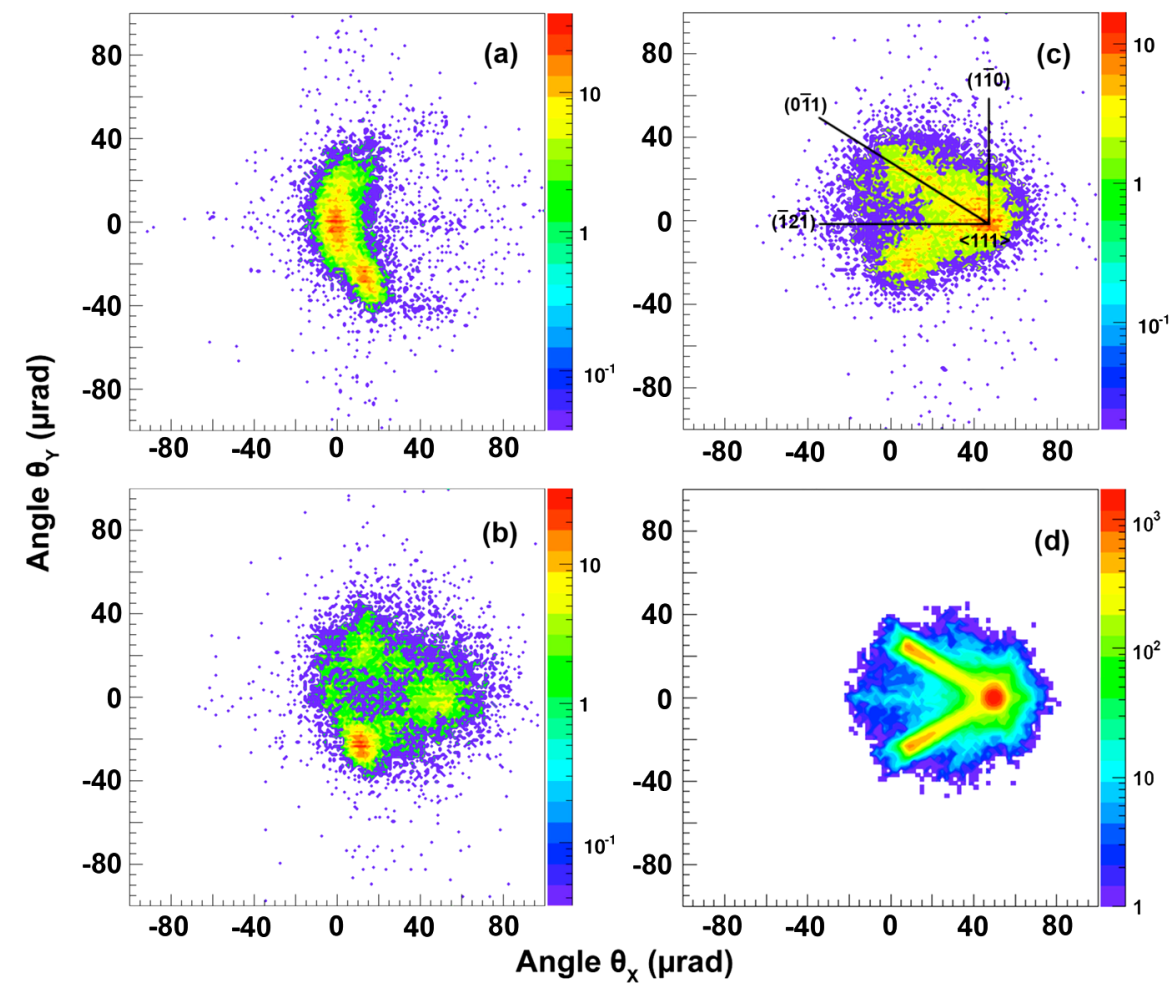

FIG. 2 (color online). Beam intensity distribution as a function of the horizontal and vertical deflections at some orientation angles $\left(\theta_{v}\right)$ of the $\langle 111\rangle$ axis with respect to the incident beam direction: (a) $\theta_{v}=-40 \mu \mathrm{rad}$, (b) $\theta_{v}=$ $-15 \mu \mathrm{rad}$, and (c) $\theta_{v}=0 \mu \mathrm{rad}$. The beam particles shown in the figure are only those hitting the entry face of the crystal and satisfying the condition $\left(\sigma_{x}^{2}+\sigma_{y}^{2}\right)<(5 \mu \mathrm{rad})^{2}$, where $\sigma_{x}$ and $\sigma_{y}$ are the horizontal and vertical values, respectively, of the single particle divergence at the crystal position. (d) Simulation of experimental conditions in case of perfect alignment of the $\langle 111\rangle$ axis with the beam direction, for comparison with the experimental case (c). 


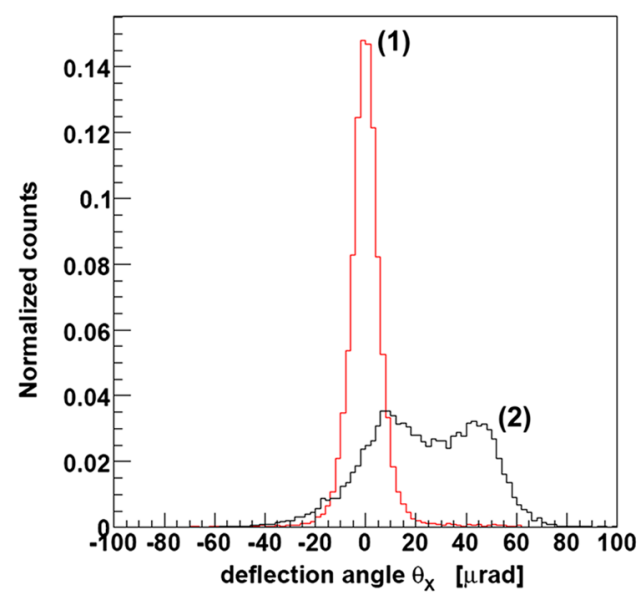

FIG. 3 (color online). Projection on the horizontal plane of the unperturbed beam profile (curve 1) and of the distribution shown in Fig. 2(c) (curve 2). Each distribution is normalized to unity. The figure highlights that most of the particles in the incoming beam were shifted apart by significant angles through interaction with the crystal.

clearly observed, formed by deflection of particles due to axial channeling (rightmost spot) and channeling in skew planar channels (two symmetric spots on the left), as predicted by simulation [10]. In contrast to planar channelling, there is no beam near the incident beam direction in the distribution.

Figure 2(d) shows the angular distribution for $400 \mathrm{GeV} / c$ protons deflected at a perfect alignment with the $\langle 111\rangle$ axis of a bent silicon crystal simulated for the same conditions of the experiment. The model of atomicstring lattice [15] with the atomic potential and electron density obtained in the Doyle-Turner approximation for the atomic scattering factors was used for simulation. The simulation results allowed one to estimate the contribution of hyperchanneled particles to the distribution peak near the full bending angle. This contribution is smaller than $2 \%$ even for a perfect alignment of the crystal axis with the beam.

The horizontal projection of the experimental distribution for almost perfect axial alignment [Fig. 2(c)] is shown in curve 2 of Fig. 3 as compared to the unperturbed condition (curve 1). Deflection efficiency due to AC can be conservatively estimated through the fraction of particles whose horizontal deflection angles exceed $40 \mu \mathrm{rad}$. The particles with smaller deflection angles were dechanneled or captured by the skew channels with high probability. Deflection efficiency for AC was about 30\%; i.e., beam deflection through doughnut scattering allowed us to significantly enhance the number of deflected particles.
In summary, deflection of high-energy protons via axial channeling in a bent crystal, mainly driven by the mechanism of doughnut scattering, was clearly observed. Hyperchanneling provides only a small contribution $\sim 1 \%$ to the deflection efficiency. Summing up the contribution of doughnut scattering and of planar channeling in skew planes (which is a by-product of AC), it comes out that about $90 \%$ of the particles are deflected by the crystal (see Fig. 2). Therefore, a short bent crystal in AC mode can be considered for using in the collider beam collimation system as a primary collimator, in addition to planar channeling and volume reflection modes of the crystals, since most of the particles interacting with the crystal are deflected with sufficiently large angles to be directed deep onto the secondary collimator.

We acknowledge partial support by the European Community-Research Infrastructure Activity under the FP6 Structuring the European Research Area program (CARE, Contract No. RII3-CT-2003-506395), the INFN NTA-HCCC, the INTAS Grant No. 05-103-7525 and MIUR 2006028442 project, Russian Foundation for Basic Research Grants No. 06-02-16912 and No. 08-0291020, RF President Foundation Grant No. SS-3057-20062, Program "Elementary Particle Physics and Fundamental Nuclear Physics" of the Russian Academy of Sciences.

[1] J. Lindhard, K. Dan. Vidensk. Selsk. Mat. Fys. Medd. 34, 14 (1965).

[2] R. A. Carrigan et al., Phys. Rev. ST Accel. Beams 1, 022801 (1998).

[3] A. S. Denisov et al., Nucl. Instrum. Methods Phys. Res., Sect. B 69, 382 (1992).

[4] N. Doble et al., Nucl. Instrum. Methods Phys. Res., Sect. B 119, 181 (1996).

[5] A. G. Afonin et al., Phys. Rev. Lett. 87, 094802 (2001).

[6] S. Bellucci et al., Phys. Rev. Lett. 90, 034801 (2003).

[7] A. M. Taratin and S. A. Vorobiev, Pis'ma Zh. Tekh. Fiz. 4, 947 (1978).

[8] J. F. Bak et al., Nucl. Phys. B242, 1 (1984).

[9] A. Baurichter et al., Nucl. Instrum. Methods Phys. Res., Sect. B 119, 172 (1996).

[10] A. A. Greenenko and N. F. Shul'ga, Phys. Lett. B 454, 161 (1999).

[11] N. F. Shul'ga and A. A. Greenenko, Phys. Lett. B 353, 373 (1995).

[12] S. Baricordi et al., Appl. Phys. Lett. 91, 061908 (2007).

[13] W. Scandale et al., Rev. Sci. Instrum. 79, 023303 (2008).

[14] A. M. Frolov et al., Nucl. Instrum. Methods 178, 319 (1980).

[15] A. M. Taratin and S. A. Vorobiev, Phys. Lett. A 115, 401 (1986). 